\title{
Model-based discrete relaxation process representation of band-limited power-law attenuation
}

\author{
Sven Peter Näsholm \\ Department of Informatics, University of Oslo, P. O. Box 1080, NO-0316 Oslo, Norway
}

(Dated: November 16, 2012)

\begin{abstract}
Frequency-dependent acoustical loss due to a multitude of physical mechanisms is commonly modeled by multiple relaxations. For discrete relaxation distributions, such models correspond with causal wave equations of integer-order temporal derivatives. It has also been shown that certain continuous distributions may give causal wave equations with fractional-order temporal derivatives. This paper demonstrates analytically that if the wave-frequency $\omega$ satisfies $\Omega_{\mathrm{L}} \ll \omega \ll \Omega_{\mathrm{H}}$, a continuous relaxation distribution populating only $\Omega \in\left[\Omega_{\mathrm{L}}, \Omega_{\mathrm{H}}\right]$ gives the same effective wave equation as for a fully populated distribution. This insight sparks the main contribution: the elaboration of a method to determine discrete relaxation parameters intended for mimicking a desired attenuation behavior for band-limited waves. In particular, power-law attenuation is discussed as motivated by its prevalence in complex media, e.g. biological tissue. A Mittag-Leffler function related distribution of relaxation mechanisms has previously been shown to be related to the fractional Zener wave equation of three power-law attenuation regimes. Because these regimes correspond to powerlaw regimes in the relaxation distribution, the idea is to sample the distribution's compressibility contributions evenly in logarithmic frequency while appropriately taking the stepsize into account. This work thence claims to provide a model-based approach to determination of discrete relaxation parameters intended to adequately model attenuation power-laws.
\end{abstract}

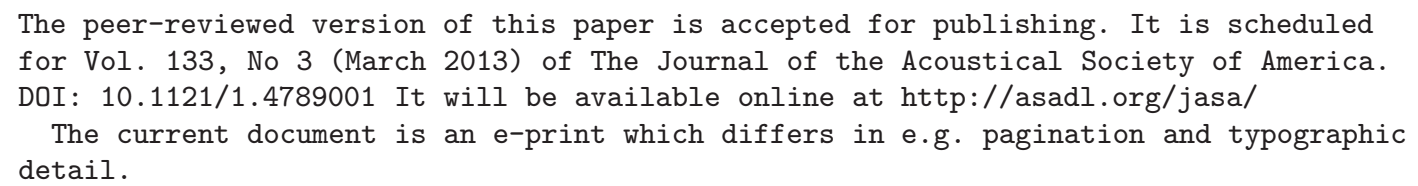

PACS numbers: 43.80.Cs, 43.20.Hq, 43.20.Jr, 43.20.Bi

\section{INTRODUCTION}

This paper concerns the determination of multiple relaxation parameters.

It is empirically observed that attenuation in biological tissue and other complex media such as polymers, rocks, and rubber often follows a power-law in frequency: $\alpha_{k}(\omega) \propto \omega^{\eta}$. with the exponent between 0 and 2 (Szabo and $\mathrm{Wu}(2000))$. Such power-laws can be valid over many frequency decades. For acoustic modeling, timefractional derivative wave equations have been shown to imply power-law attenuation over wide frequency bands (Holm and Sinkus (2010); Holm and Näsholm (2011)). Such fractional wave equations can be obtained from linearized conservation of mass and momentum in combination with time-fractional constitutive relations connecting stress and strain. Associated nonlinear fractional wave equations are presented in Prieur and Holm (2011) and Prieur et al. (2012) while related linear wavepropagation simulations are reported, e.q. in Wismer and Ludwig (1995); Liebler et al. (2004); Wismer (2006); Caputo et al. (2011).

Moreover, the multiple relaxation mechanism framework of Nachman et al. (1990) is widely considered adequate for acoustic wave modeling in lossy complex media like those encountered in medical ultrasound. It relies on thermodynamics and first principles of acoustical physics.
The corresponding lossy wave equation for $N$ discrete relaxation mechanisms is a partial differential equation with its highest time derivative of order $N+2$. This model is in the following denoted the Nachman-SmithWaag (NSW) model.

Viscoelastic constitutive stress-strain models are generally possible to convert into a Maxwell-Wiechert description with springs and dashpots in parallel. The NSW model is linked to fractional derivative modeling in Näsholm and Holm (2011), where a continuum of relaxation mechanisms is assumed. The compressibility contributions were assumed to be distributed following a function related to the Mittag-Leffler function. It was shown that the wave equation corresponding to this distribution is identical to the fractional Zener wave equation. Actually, a Maxwell-Wiechert description of the fractional Zener stress-strain relation was thereby implicitly verified. In Adolfsson et al. (2005), a very large number of weighted Maxwell elements evenly distributed in the linear frequency scale are shown to give the same stress response as a fractional order viscoelastic model. We also note that the rheological fractional spring-pot element was interpreted in terms of weighted springs and dashpots in Papoulia et al. (2010).

Kelly and McGough (2009) demonstrated that hierarchical fractal ladder networks of springs and dashpots can lead to power-law attenuation in a low-frequency regime. 
This approach however requires a large number of degrees of freedom which makes parameter fits cumbersome.

Band-limited fits to power-law acoustic attenuation for relaxation models with $N=2$ and 3 are exemplified by Tabei et al. (2003) and Yang and Cleveland (2005). In the latter, one of the mechanisms is assumed to be of very short relaxation frequency, thus representing a thermoviscous component. The two other mechanism relaxation frequencies, the corresponding two compressibility contributions, and the compressibility of the thermoviscous component are determined through numerical minimization of the difference between the resulting attenuation and the desired power-law.

For a large number of modeled relaxation mechanisms, such numerical optimization of the parameter fit turns very intricate.

Recently published works which stress the need for straightforward and accurate determination of discrete relaxation representations that generate power-law attenuation, are e.g. Roitner et al. (2012) (see Section II) and Treeby et al. (2012) (see Section II.C). See also the introduction in Liebler et al. (2004).

The present paper establishes a systematic modelbased method to choose the compressibility contribution and the relaxation frequency for each of $N$ relaxation processes, to model power-law attenuation over a given wave frequency band. This discrete parameter selection method is based on the continuous distribution of relaxation processes previously shown to result in fractional Zener wave equation, which in turn generates 3 distinctive power-law attenuation regimes Näsholm and Holm (2011)). The present work also studies analytically the effect of letting this distribution cover only a limited frequency band.

This paper is organized as follows. The Theory Section II first reviews and partially extends relevant NSW theory, fractional Zener model considerations, and the link between those. Then it presents new developments related to band-limited continuous and discrete relaxation distributions, as well as the related attenuation for waves of frequencies within and outside the populated relaxation bandwidth. Section II.E is central because it describes how to select discrete relaxation process parameters to get approximate power-law attenuation over a given wave-frequency band. Section III provides two numerical examples exemplifying how discrete relaxation parameters may be determined in order to attain powerlaw attenuation. Discussions and conclusions are given in Section IV

\section{THEORY}

\section{A. Relaxation processes modeling within the NSW framework}

\section{Discrete relaxation distribution}

The NSW model of multiple discrete relaxation processes results in the frequency-domain generalized com- pressibility (Nachman et al. $($ (1990))

$$
\kappa(\omega)=\kappa_{0}-i \omega \sum_{\nu=1}^{N} \frac{\kappa_{\nu} \tau_{\nu}}{1+i \omega \tau_{\nu}}=\kappa_{0}-i \omega \sum_{\nu=1}^{N} \frac{\kappa_{\nu}}{\Omega_{\nu}+i \omega},
$$

where the mechanisms $\nu=1 \ldots N$, have the relaxation times $\tau_{1}, \ldots, \tau_{N}$ and the compressibility contributions $\kappa_{1}, \ldots, \kappa_{N}$. In the present work $\Omega_{\nu} \triangleq 1 / \tau_{\nu}$ denotes the process relaxation frequency.

The frequency-domain generalized compressibility is in the following simply denoted compressibility. In some branches of science it is instead called complex compliance $J^{*}(\omega)$, being defined as the ratio between strain and stress: $\kappa(\omega) \triangleq \epsilon(\omega) / \sigma(\omega)$. It is hence directly related to the constitutive stress-strain relation.

\section{Continuous relaxation distribution}

Following Näsholm and Holm (2011), a representation of Eq. (1) when considering a continuum of relaxation mechanisms distributed in the frequency band $\Omega \in\left[\Omega_{\mathrm{L}}, \Omega_{\mathrm{H}}\right]$ with the compressibility contributions described by the distribution $\widetilde{\kappa}_{\nu}(\Omega)$ becomes

$$
\kappa_{\mathrm{N}}(\omega) \triangleq \kappa_{0}-i \omega \int_{\Omega_{\mathrm{L}}}^{\Omega_{\mathrm{H}}} \frac{\tilde{\kappa}_{\nu}(\Omega)}{\Omega+i \omega} \mathrm{d} \Omega .
$$

Letting the integral go from $\Omega_{\mathrm{L}}=0$ to $\Omega_{\mathrm{H}}=\infty$, and instead incorporating any possible relaxation distribution bandwidth limitation of $\tilde{\kappa}_{\nu}(\Omega)$ into the distribution, we define $\kappa_{\nu}(\Omega) \triangleq H\left(\Omega-\Omega_{\mathrm{L}}\right) H\left(\Omega_{\mathrm{H}}-\Omega\right) \tilde{\kappa}_{\nu}(\Omega)$, where $H(\Omega)$ denotes the Heaviside step function. Then the integral (2) is a Stieltjes transform. By application of the Laplace transform relation, which for real $\omega$, real $t$, and real $\Omega$ is valid for $\Omega>0$ :

$$
\mathcal{L}_{t}\left\{H(t) e^{-i \omega t}\right\}(\Omega) \triangleq \int_{0}^{\infty} H(t) e^{-i \omega t} e^{-\Omega t} \mathrm{~d} t=\frac{1}{\Omega+i \omega}
$$

from the $t$ domain to the $\Omega$ domain, the compressibility (2) may be written as

$$
\kappa_{\mathrm{N}}(\omega)=\kappa_{0}-i \omega \int_{0}^{\infty} \kappa_{\nu}(\Omega)\left\{\int_{0}^{\infty} H(t) e^{-\Omega t} e^{-i \omega t} \mathrm{~d} t\right\} \mathrm{d} \Omega,
$$

which by virtue of Fubini's theorem may be written as

$$
\begin{aligned}
\kappa_{\mathrm{N}}(\omega) & =\kappa_{0}-i \omega \int_{0}^{\infty} e^{-i \omega t}\left\{H(t) \int_{0}^{\infty} \kappa_{\nu}(\Omega) e^{-\Omega t} \mathrm{~d} \Omega\right\} \mathrm{d} t \\
& =\kappa_{0}-i \omega \mathcal{F}_{t}\left\{H(t) \mathcal{L}_{\Omega}\left\{\kappa_{\nu}(\Omega)\right\}(t)\right\}(\omega),
\end{aligned}
$$

where the Fourier transform from the $t$ domain to the $\omega$ domain is $\mathcal{F}_{t}\{f(t)\}(\omega) \triangleq \int_{-\infty}^{\infty} e^{-i \omega t} f(t) \mathrm{d} t$. 
Another equivalent representation of the compressibility (2) is

$\kappa_{\mathrm{N}}(\omega)=\kappa_{0}-\omega^{2} \int_{0}^{\infty} \frac{\kappa_{\nu}(\Omega)}{\Omega^{2}+\omega^{2}} \mathrm{~d} \Omega-i \omega \int_{0}^{\infty} \frac{\Omega \kappa_{\nu}(\Omega)}{\Omega^{2}+\omega^{2}} \mathrm{~d} \Omega$,

which is found by multiplying both the numerator and the denominator of Eq. (2) by $(\Omega-i \omega)$.

\section{Attenuation and phase velocity}

The conventional decomposition of the frequencydependent wavenumber $k(\omega)$ into its real and imaginary parts, gives the phase velocity $c_{p}(\omega)=\omega / \Re\{k(\omega)\}$ and the attenuation $\alpha_{k}(\omega)=-\Im\{k(\omega)\}$.

Combining the definition of $\kappa(\omega) \triangleq \epsilon(\omega) / \sigma(\omega)$ with the linearized conservation of mass and momentum (see Näsholm and Holm (2011) and the references therein for details) gives

$$
k^{2}(\omega)=\omega^{2} \rho_{0} \kappa(\omega) .
$$

In general, the attenuation and the phase velocity are thus given from the dispersion relation above as

$$
\begin{aligned}
& \alpha_{k}(\omega)=-\Im\{k\}=-\omega \sqrt{\rho_{0}} \Im\{\sqrt{\kappa(\omega)}\} \text { and } \\
& c_{p}(\omega)=\omega / \Re\{k\}=\rho_{0}^{-1 / 2} / \Re\{\sqrt{\kappa(\omega)}\} .
\end{aligned}
$$

Analyzing this expression further, we see that in the small-attenuation regime where the $\kappa_{0}$ part of (7) dominates over the imaginary part infers

$$
\begin{aligned}
& \sqrt{\kappa_{\mathrm{N}}(\omega)} \\
& =\sqrt{\kappa_{0}-\omega^{2} \int_{0}^{\infty} \frac{\kappa_{\nu}(\Omega)}{\Omega^{2}+\omega^{2}} \mathrm{~d} \Omega-i \omega \int_{0}^{\infty} \frac{\Omega \kappa_{\nu}(\Omega)}{\Omega^{2}+\omega^{2}} \mathrm{~d} \Omega} \\
& \approx \sqrt{\kappa_{0}}-\frac{1}{2 \sqrt{\kappa_{0}}}\left[-\omega^{2} \int_{0}^{\infty} \frac{\kappa_{\nu}(\Omega)}{\Omega^{2}+\omega^{2}} \mathrm{~d} \Omega-i \omega \int_{0}^{\infty} \frac{\Omega \kappa_{\nu}(\Omega)}{\Omega^{2}+\omega^{2}} \mathrm{~d} \Omega\right.
\end{aligned}
$$

Then the attenuation $\alpha_{k}$ is given from the approximation

$$
\begin{aligned}
\alpha_{k}(\omega)= & -\omega \sqrt{\rho_{0}} \Im\left\{\sqrt{\kappa_{\mathrm{N}}(\omega)}\right\} \\
& \approx A \omega^{2} \int_{0}^{\infty} \frac{\Omega \kappa_{\nu}(\Omega)}{\Omega^{2}+\omega^{2}} \mathrm{~d} \Omega \\
& =A \omega^{2} \int_{0}^{\infty} \int_{0}^{\infty} \kappa(\Omega) e^{-\Omega t} \cos (\omega t) \mathrm{d} t \mathrm{~d} \Omega \\
& =A \omega^{2} \mathcal{F}_{\mathrm{c}}\left\{\mathcal{L}_{\Omega}\left\{\kappa_{\nu}(\Omega)\right\}(t)\right\}(\omega),
\end{aligned}
$$

where $\mathcal{F}_{\mathrm{c}}$ denotes the Fourier cosine transform from the $t$ domain to the $\omega$ domain, and $\mathcal{L}_{\Omega}$ denotes the Laplace transform from the $\Omega$ domain to the $t$ domain. The introduced $A$ is a frequency-independent scalar. The integral of Eq. (11) is a Widder potential transform (Widder $(1966))$.

Using Eq. (9) and (12) under the small-attenuation constraint, the relaxation process spectrum $\widetilde{\kappa}_{\nu}(\Omega)$ corresponding to some frequency-dependent attenuation model $\widetilde{\alpha}_{k}(\omega)$ may hence be constructed using the inverse transform recipe

$$
\widetilde{\kappa}_{\nu}(\Omega)=A \cdot \mathcal{L}_{t}^{-1}\left\{\mathcal{F}_{\mathrm{c}}^{-1}\left\{\frac{\widetilde{\alpha}_{k}(\omega)}{\omega^{2}}\right\}(t)\right\}(\Omega) .
$$

Expression (13) is similar to what was found using an approach reported in Vilensky et al. (2012), however a formula equivalent to (11) was used alreadv in e.q. Pauly and Schwan (1971). The small-attenuation assumption is probably reasonable for compressional wave propagation in biological tissue. By contrast, for shear-wave propagation, the attenuation is generally much more pronounced (Szabo and Wu (2000)).

For a discrete set of relaxation mechanisms under the small-approximation assumption, we see from Eq. (11) that the total attenuation is just the sum of the contribution from each mechanism: $\alpha_{k}(\omega)=\sum_{\nu=1}^{N} \alpha_{\nu}(\omega)$, where the contribution of mechanism $\nu$ is given by

$$
\alpha_{\nu}(\omega)=\frac{\kappa_{\nu} \Omega_{\nu} \omega^{2}}{\omega^{2}+\Omega_{\nu}^{2}} .
$$

Such behavior is especially well documented in air (Bass et al. (1995)) and in sea water (Ainslie and McColm (1998)).

\section{B. The fractional Zener wave equation}

As a consequence of the fractional Zener model stressstrain relation (see e.g. Bagley and Torvik (1983)), the frequency-domain fractional Zener compressibility is obtained from the ratio $\epsilon(\omega) / \sigma(\omega)$ :

$$
\kappa_{Z}(\omega) \triangleq \kappa_{0} \frac{1+\left(\tau_{\epsilon} i \omega\right)^{\beta}}{1+\left(\tau_{\sigma} i \omega\right)^{\alpha}} .
$$

Due to thermodynamic constraints, $0<\alpha \leq 1$ and $\beta \leq \alpha$ ](Glöckle and Nonnenmacher (1991)), however the case $\alpha=\beta$ is the most well-behaved from a physical point of view Rossikhin and Shitikova (2001). Insertion of the compressibility (15) into the dispersion relation (8), generates the fractional Zener dispersion relation (Holm and Näsholm (2011))

$$
k^{2}=\frac{\omega^{2}}{c_{0}^{2}} \frac{1+\left(\tau_{\epsilon} i \omega\right)^{\beta}}{1+\left(\tau_{\sigma} i \omega\right)^{\alpha}} .
$$

This is the spatio-temporal frequency domain representation of the five-parameter fractional Zener wave equation which by inverse transform hence becomes

$$
\nabla^{2} u-\frac{1}{c_{0}^{2}} \frac{\partial^{2} u}{\partial t^{2}}+\tau_{\sigma}^{\alpha} \frac{\partial^{\alpha}}{\partial t^{\alpha}} \nabla^{2} u-\frac{\tau_{\epsilon}^{\beta}}{c_{0}^{2}} \frac{\partial^{\beta+2} u}{\partial t^{\beta+2}}=0 .
$$

The $\alpha=\beta$ variant of the fractional Zener compressibility (15) combined with Eq. (9) results in three distinct attenuation power-laws (Holm and Näsholm (2011)):

$$
\alpha_{k} \propto \begin{cases}\omega^{1+\alpha} & \text { low-frequency regime, } \\ \omega^{1-\alpha / 2} & \text { intermediate frequency regime, } \\ \omega^{1-\alpha} & \text { high-frequency regime. }\end{cases}
$$




\section{Connecting the NSW and the fractional Zener models}

\section{The continuous relaxation distribution $\kappa_{\nu}^{\prime}(\Omega)$}

Provided that the linearized conservations of mass and momentum are valid, and given that the NSW compressibility $\kappa_{\mathrm{N}}(\omega)$ of Eq. (6) is equal to the fractional Zener compressibility $\kappa_{Z}(\omega)$ of Eq. (15), the dispersion relations from Eq. (8) are also equal. Because the dispersion relation is a spatio-temporal Fourier representation of the wave equation, $\kappa_{\mathrm{N}}(\omega)=\kappa_{\mathrm{Z}}(\omega)$ thus implies that the NSW wave equation becomes equal to the fractional Zener wave equation (17). As brought forward in Näsholm and Holm (2011), there exists a continuous distribution $\kappa_{\nu}^{\prime}(\Omega)$ of NSW relaxation processes which implies $\kappa_{\mathrm{N}}(\omega)=\kappa_{\mathrm{Z}}(\omega)$ when $\alpha=\beta$ :

$$
\kappa_{\nu}^{\prime}(\Omega) \triangleq \frac{1}{\pi} \frac{\kappa_{0}\left(\tau_{\sigma}^{\alpha}-\tau_{\epsilon}^{\alpha}\right) \Omega^{\alpha-1} \sin (\alpha \pi)}{\left(\tau_{\sigma} \Omega\right)^{2 \alpha}+2\left(\tau_{\sigma} \Omega\right)^{\alpha} \cos (\alpha \pi)+1} .
$$

This distribution is the inverse Laplace transform of a Mittag-Leffler related expression, see Näsholm and Holm (2011) for details. Note that this link between the fractional Zener and the NSW models is valid also outside the small-attenuation regime $\Im\{k\} \ll \Re\{k\}$.

\section{2. $\kappa_{\nu}^{\prime}(\Omega)$ power-law regimes}

Below follows an analysis of the relaxation time distribution $\kappa_{\nu}^{\prime}(\Omega)$ of Eq. (19) as it evolves depending on $\Omega \tau_{\sigma}$ :

$$
\begin{aligned}
\kappa_{\nu}^{\prime}(\Omega) & =\frac{1}{\pi} \frac{\kappa_{0}\left(\tau_{\sigma}^{\alpha}-\tau_{\epsilon}^{\alpha}\right) \Omega^{\alpha-1} \sin (\alpha \pi)}{\left(\tau_{\sigma} \Omega\right)^{2 \alpha}+2\left(\tau_{\sigma} \Omega\right)^{\alpha} \cos (\alpha \pi)+1} \\
& \approx \begin{cases}C_{\mathrm{L}} \cdot \Omega^{\alpha-1}, & \text { for } \Omega \tau_{\sigma} \ll 1 \\
C_{\mathrm{I}} \cdot \Omega^{-1}, & \text { for } \Omega \tau_{\sigma} \approx 1 \\
C_{\mathrm{H}} \cdot \Omega^{-\alpha-1}, & \text { for } 1 \ll \Omega \tau_{\sigma},\end{cases}
\end{aligned}
$$

where the frequency-independent constants are:

$$
\begin{aligned}
& C_{\mathrm{L}} \triangleq \frac{\kappa_{0}\left(\tau_{\sigma}^{\alpha}-\tau_{\epsilon}^{\alpha}\right) \sin (\alpha \pi)}{\left.\pi^{\alpha}\right) \sin (\alpha \pi)}, \\
& C_{\mathrm{I}} \triangleq \frac{\kappa_{0}\left(\tau_{\sigma}^{\alpha}-\tau_{\epsilon}^{\alpha} \cos (\alpha \pi)\right)}{2 \pi \tau_{\sigma}^{\alpha}\left(1+\cos \left(1+\tau_{\epsilon}^{\alpha}\right) \sin (\alpha \pi)\right.} \\
& C_{\mathrm{H}} \triangleq \frac{\kappa_{0}\left(\tau_{\sigma}^{\alpha}-\tau_{\sigma}^{2 \alpha}\right.}{\pi \tau^{2 \alpha}}
\end{aligned}
$$

Due to the restriction $0<\alpha \leq 1$, we hence note that depending on the value of the product $\tau_{\sigma} \Omega$, the distribution $\kappa_{\nu}^{\prime}(\Omega)$ may have the form of a power-law $\kappa_{\nu}^{\prime} \propto \Omega^{d}$, where $-2<d \leq 0$.

\section{A band-limited continuum of NSW relaxation processes}

In the following, the case of a continuum of relaxation processes populating a limited frequency band $\Omega \in$ $\left[\Omega_{\mathrm{L}}, \Omega_{\mathrm{H}}\right]$ is further explored with respect to the for three different wave-frequency regimes.

\section{Formal analysis of band-limited power-law relaxation distribution}

The case of a relaxation process continuum populating the region $\Omega \in\left[\Omega_{\mathrm{L}}, \Omega_{\mathrm{H}}\right]$, for example where $\kappa_{\nu}(\Omega)$ is adequately be approximated by a power-law (see Section II.C.2 above)

$$
\kappa_{\nu}(\Omega)= \begin{cases}C \cdot \Omega^{d} & \Omega \in\left[\Omega_{\mathrm{L}}, \Omega_{\mathrm{H}}\right] \\ 0 & \text { otherwise }\end{cases}
$$

where $-2<d \leq 0$, is now investigated. Distributions of such form are related to broken power-laws, often denoted Pareto distributions, and are commonly observed in nature and society (Newman (2005)).

For $\kappa_{\nu}(\Omega)=C \cdot \Omega^{d}$, the generalized compressibility (2) may be written

$$
\kappa_{\mathrm{N}}(\omega)=\kappa_{0}-i \omega C\left[\int_{\Omega_{\mathrm{L}}}^{\infty} \frac{\Omega^{d}}{\Omega+i \omega} \mathrm{d} \Omega-\int_{\Omega_{\mathrm{H}}}^{\infty} \frac{\Omega^{d}}{\Omega+i \omega} \mathrm{d} \Omega\right]
$$

which in view of Eq. (7) and Bateman and Erdélyi (1954) [Eq. (21)], becomes

$$
\begin{aligned}
& \kappa_{\mathrm{N}}(\omega)=\kappa_{0}+\frac{C \omega^{2}}{2}\left[\Omega_{\mathrm{H}}^{d-1} \Phi\left(-\frac{\omega^{2}}{\Omega_{\mathrm{H}}^{2}}, 1, \frac{1-d}{2}\right)\right. \\
& \left.-\Omega_{\mathrm{L}}^{d-1} \Phi\left(-\frac{\omega^{2}}{\Omega_{\mathrm{L}}^{2}}, 1, \frac{1-d}{2}\right)\right] \\
& +\frac{i C \omega}{2}\left[\Omega_{\mathrm{H}}^{d} \Phi\left(-\frac{\omega^{2}}{\Omega_{\mathrm{H}}^{2}}, 1,-\frac{d}{2}\right)-\Omega_{\mathrm{L}}^{d} \Phi\left(-\frac{\omega^{2}}{\Omega_{\mathrm{L}}^{2}}, 1,-\frac{d}{2}\right)\right],
\end{aligned}
$$

where $\Phi(x, \nu, u)$ is the analytic continuation of the Lerch transcendent. In Section II.D.2 below, the compressibility (24) is further analyzed. First a continuous bandlimited power-law relaxation spectrum is considered and the corresponding generalized compressibility is deduced. Then three different wave-frequency regimes are considered for any band-limited relaxation relaxation $\kappa_{\nu}(\Omega)$ and the corresponding attenuation functions are brought forth. For $\nu=1$, the function $\Phi(x, \nu, u)$ is related to the analytic continuation of the Gauss hypergeometric function as: (Bateman and Erdélyi (1954), Eq. (1.10))

$$
\Phi(x, 1, u)=u_{2}^{-1} F_{1}(1, u ; 1+u ; x),
$$

to which there is a direct connection to fractional calculus because (Samko et al. (1993))

$$
{ }_{2} F_{1}(a, b ; c ; x)=\frac{\Gamma(a) x^{1-c}}{\Gamma(b)}{ }_{0} D_{x}^{1-c} \frac{x^{b-1}}{(1-x)^{a}} .
$$

For practical purposes when $\omega$ is far from the relaxation process cut-off frequencies $\Omega_{\mathrm{L}}$ and $\Omega_{\mathrm{H}}$, it is however not necessary to evaluate the Lerch transcendents of (24) to find $\kappa(\omega)$ and hence determine the frequencydependent attenuation and phase velocity. The three relevant frequency regimes are considered in the following two subsections. 


\section{The $\Omega_{L} \ll \omega \ll \Omega_{H}$ frequency range for any $\kappa_{\nu}(\omega)$}

In case the wave-frequency is within the frequency band of the relaxation processes, in the integrals of (7) we change the integration limits from 0 into $\Omega_{\mathrm{L}}$ and from $\infty$ into $\Omega_{\mathrm{H}}$ and in addition make the variable change $u \triangleq \Omega / \omega$ to result in

$\kappa_{\mathrm{N}}(\omega)=\kappa_{0}-\omega \int_{\frac{\Omega_{\mathrm{L}}}{\omega}}^{\frac{\Omega_{\mathrm{H}}}{\omega}} \frac{\kappa_{\nu}(u \omega)}{u^{2}+1} \mathrm{~d} u-i \omega \int_{\frac{\Omega_{\mathrm{L}}}{\omega}}^{\frac{\Omega_{\mathrm{H}}}{\omega}} \frac{u \kappa_{\nu}(u \omega)}{u^{2}+1} \mathrm{~d} u$.

For the analyzed case $\Omega_{\mathrm{L}} \ll \omega \ll \Omega_{\mathrm{H}}$, the integration limits then become $\Omega_{\mathrm{L}} / \omega \rightarrow 0$ and $\Omega_{\mathrm{H}} / \omega \rightarrow \infty$. Then performing the variable change back into $\Omega=u \omega$ makes it clear that under the given conditions the effective compressibility thus becomes equal to the compressibility related to the fully-populated relaxation frequency band (17). A similar analysis may also be done directly in (2). The fractional Zener wave equation (17) and its associated attenuation and phase velocity expressions (see Näsholm and Holm (2011)) are consequently valid also for the band-limited distribution of relaxation processes, as long as the wave frequency $\omega$ is much lower than $\Omega_{\mathrm{H}}$ and much greater than $\Omega_{\mathrm{L}}$.

Interestingly, the analysis above prompts the conclusion that for a band-limited $\left(\Omega_{\mathrm{L}} \ll \omega \ll \Omega_{\mathrm{H}}\right)$ powerlaw relaxation distribution $\kappa_{\nu}(\Omega) \propto \Omega^{d}$, which results in the compressibility $\kappa_{\mathrm{N}}(\omega)$ given by (24), there is a direct relation to the attenuation power-law valid within $\Omega_{\mathrm{L}} \ll \omega \ll \Omega_{\mathrm{H}}$ :

First, consider the low-frequency power-law regime of the distribution $\kappa_{\nu}^{\prime}(\Omega)$ in Eq. (19) being populated in the limited relaxation frequency band. Then $\kappa_{\nu}^{\prime}(\Omega) \propto \Omega^{d}$ which gives $d=(\alpha-1) \in[-1,0]$, because $\alpha \in[0,1]$. By virtue of Eq. (18) this corresponds to the attenuation power-law $\alpha_{k}(\omega) \propto \omega^{\eta}$ with the exponent $\eta \in[1+\alpha] \Leftrightarrow$ $\eta \in[1,2]$. Second, consider instead the high-frequency regime of the distribution $\kappa_{\nu}^{\prime}(\Omega)$ being populated in the limited relaxation frequency band. This leads to $\kappa_{\nu}^{\prime}(\Omega) \propto$ $\Omega^{d}$ with $d=(-\alpha-1) \in[-2,-1]$.

Hence a band $\Omega_{\mathrm{L}} \ll \omega \ll \Omega_{\mathrm{H}}$ populated with relaxation frequency contributions $\kappa_{\nu}(\Omega) \propto \Omega^{d}$ gives a bandlimited power-law attenuation $\alpha_{k}(\omega) \propto \omega^{d+2}$ both for $d \in[-2,-1]$ and for $d \in[-1,0]$.

3. The $\omega \ll \Omega_{L}<\Omega_{H}$ and $\Omega_{L}<\Omega_{H} \ll \omega$ frequency regimes for any $\kappa_{\nu}(\omega)$

As pointed out in Näsholm and Holm (2011), the case of the wave-frequency being much lower than the populated relaxation process frequency band $\Omega \in\left[\Omega_{\mathrm{L}}, \Omega_{\mathrm{H}}\right]$, an analysis similar to the LF limit of Nachman et al. (1990) results in $\alpha_{k} \propto \omega^{2}$. By the same token, for the wavefrequency being much higher than the populated relaxation proportional to frequency band, the attenuation is frequency-independent.

Within these frequency regimes, the effective wave equation is hence the same as for a single discrete relaxation mechanism with the compressibility contribution being proportional to the impulse $\delta\left(\Omega-\left(\Omega_{\mathrm{L}}+\Omega_{\mathrm{H}}\right) / 2\right)$. This is similar as for a medium with one single discrete NSW relaxation process.

\section{E. $N$ discrete NSW relaxation processes to get band-limited attenuation power-law}

Based on the developments in Section II.D, here a method is elaborated to determine the $\Omega_{\nu}$ and $\kappa_{\nu}$ parameters of a discrete set of $N$ relaxation processes intended to result in an appropriate attenuation power-law $\alpha_{k}(\omega) \propto \omega^{\eta}$ for waves within the bandwidth $\omega \in\left[\Omega_{\mathrm{L}}, \Omega_{\mathrm{H}}\right]$. The power-law exponent satisfies $\eta \in[0,2]$.

First, based on (18), the $\eta$ exponent determines whether a low, high, or intermediate frequency model is applied. Based on this, $\tau_{\sigma}$ is then set so that $1 / \tau_{\sigma}$ is either much higher or much lower than the frequency region of interest $\left[\Omega_{\mathrm{L}}, \Omega_{\mathrm{H}}\right]$. Subsequently, the relaxation process frequencies $\Omega_{\nu}$ are sampled within $\left[\Omega_{\mathrm{L}}, \Omega_{\mathrm{H}}\right]$, equispaced in the $\log \Omega$ domain hence giving

$$
\Omega_{\nu}=\Omega_{\mathrm{L}}^{\frac{N-\nu}{N-1}} \Omega_{\mathrm{H}}^{\frac{\nu-1}{N-1}} .
$$

Thereafter, the compressibility contribution $\kappa_{\nu}\left(\Omega_{\nu}\right)$ of each process is decided from $\kappa_{\nu}^{\prime}\left(\Omega_{\nu}\right)$ of (19). Alternatively, the relevant power-law approximate expression of (20) may be chosen. Finally, the $\tau_{\epsilon}$ parameter is adjusted to achieve the attenuation $\alpha_{k}\left(\omega_{\text {ref }}\right)=\alpha_{\text {ref }}$ at some appropriate reference frequency $\omega_{\text {ref }} \in\left[\Omega_{\mathrm{L}}, \Omega_{\mathrm{H}}\right]$.

For calculation of the resulting compressibility $\kappa_{\mathrm{N}}(\omega)$, the discretized approximation of the integral (2) must take into account the uneven stepsize

$$
\Delta \Omega_{\nu}=\Omega_{\nu}\left(1-\left(\Omega_{\mathrm{L}} / \Omega_{\mathrm{H}}\right)^{1 /(N-1)}\right) .
$$

The $\kappa_{N}(\omega)$ estimate $\hat{\kappa}_{N}(\omega)$ which approximates (2) for the chosen discrete relaxation process parameters is hence

$$
\hat{\kappa}_{\mathrm{N}}(\omega)=\kappa_{0}-i \omega \sum_{\nu=1}^{N} \frac{\kappa_{\nu}\left(\Omega_{\nu}\right)}{\Omega_{\nu}+i \omega} \Delta \Omega_{\nu}
$$

From this compressibility, the attenuation and phase velocity may be calculated in the conventional manner using Eq. (9). The attenuation resulting from this relaxation process parameter decision approach is explored in the following 2 numerical examples.

\section{NUMERICAL EXAMPLES}

\section{A. Power-law $\alpha_{k} \propto \omega^{1.1}$ for $f \in[100 \mathrm{kHz}, 30 \mathrm{MHz}]$}

Below follows an explicit example of modeling an attenuation power-law $\alpha_{k} \propto \omega^{1.1}$ between $100 \mathrm{kHz}$ and $30 \mathrm{MHz}$ using discrete relaxation processes. Such attenuation is relevant for medical ultrasound imaging. The chosen medium properties, which are listed in Table \ are the same as in Yang and Cleveland (2005). The attenuation resulting from the parameter selection approach 
TABLE I. Medium parameters for the attenuation power-law fit of Section III.A similar to the Yang and Cleveland (2005) parameters.

\begin{tabular}{lr}
\hline \hline Equilibrium speed of sound, $c_{0}(\mathrm{~m} / \mathrm{s})$ & 1540 \\
Density, $\rho_{0}\left(\mathrm{~kg} / \mathrm{m}^{3}\right)$ & 1050 \\
Zero-freq. compressibility, $\kappa_{0}=\frac{1}{c_{0}^{2} \rho_{0}}\left(\mathrm{~Pa}^{-1}\right)$ & $4.0158 \cdot 10^{-10}$ \\
Wanted attenuation at $1 \mathrm{MHz}, \alpha_{0}(\mathrm{~dB} / \mathrm{MHz} / \mathrm{cm})$ & 0.3 \\
Wanted attenuation power-law exponent, $\eta$ & 1.1 \\
\hline \hline
\end{tabular}
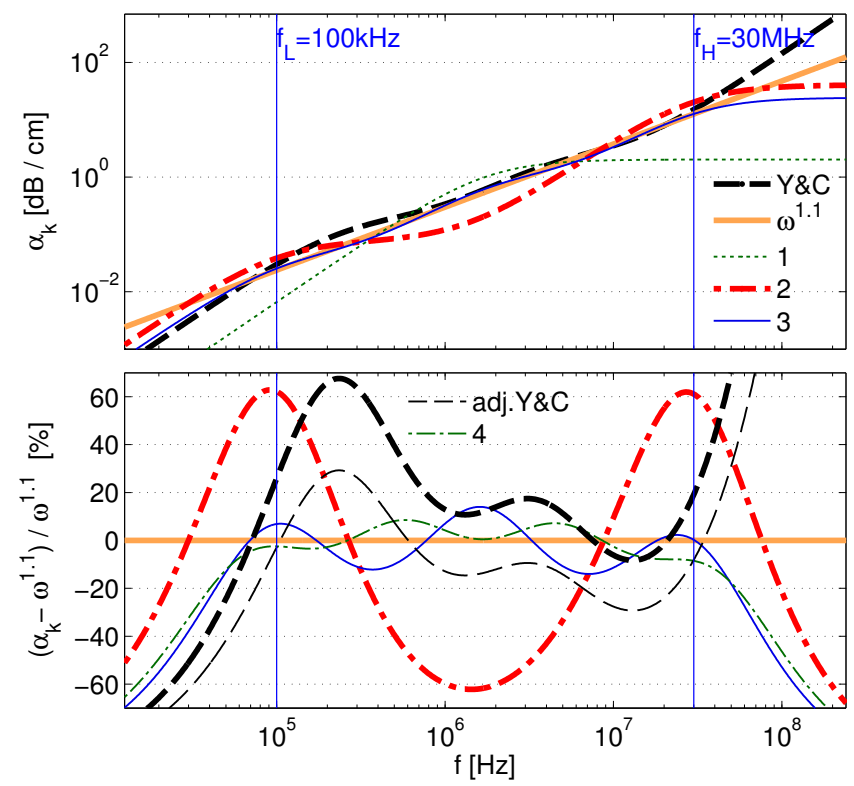

FIG. 1. (Color online) Attenuation as a function of frequency for $N$ o-relaxation mechanisms determined by the method proposed in Section \I.E aiming at constructing a power-law valid within $100 \mathrm{kHz}$ and $30 \mathrm{MHz}$. Also displayed are the Yang and Cleveland (2005) $N=3$ attenuation and an ideal power-law $\propto \omega^{1.1}$ (thick solid line). Table凹lists the medium properties. Top pane: Attained attenuations. Bottom pane: Relative difference between the attained relaxation attenuation functions and the power-law. The horizontal axes represent wave-frequency. For visualization convenience, each attenuation is normalized in order to make the minimum and maximum relative differences equal in magnitude within the relevant frequency interval. The Yang and Cleveland attenuation is included both with normalization (thick dashed line) and without (thin dashed line, only included in the bottom pane). The attenuation is shown for $N=1$ (thin dashed line), $N=2$ (thick dash-dotted line), and $N=3$ (thin solid line). The relaxation parameters $\Omega_{\nu}$ and $\kappa_{\nu}$ are displayed in Table II

proposed in Section II.E, was applied for $N=1, \ldots, 4$ relaxation mechanisms using $\Omega_{\mathrm{L}}=100 \mathrm{kHz}$ and $\Omega_{\mathrm{H}}=$ $10 \mathrm{MHz}$. The consequent attenuation functions as calculated from the NSW compressibility in Eq. (1) are displayed in Fig. 1. where also a pure $\alpha_{k}(\omega) \propto \omega^{1.1}$ law is plotted, as well as the Yang and Cleveland attenuation. In Yang and Cleveland (2005), a numerical least squares
TABLE II. Relaxation process frequencies $\Omega_{\nu}$ and compressibility contributions $\kappa_{\nu}$ corresponding to the attenuation functions displayed in Fig 1. The parameters were attained using the Section II.E method for $N=1,2,3$ relaxation mechanisms.

\begin{tabular}{l|ccc|ccc}
\hline \hline & $\begin{array}{c}\Omega_{1} \\
(\mathrm{MHz})\end{array}$ & $\begin{array}{c}\Omega_{2} \\
(\mathrm{MHz})\end{array}$ & $\begin{array}{c}\Omega_{3} \\
(\mathrm{MHz})\end{array}$ & $\begin{array}{c}\kappa_{1} \\
(\mathrm{TPa})^{-1}\end{array}$ & $\begin{array}{c}\kappa_{2} \\
(\mathrm{TPa})^{-1}\end{array}$ & $\begin{array}{c}\kappa_{3} \\
(\mathrm{TPa})^{-1}\end{array}$ \\
\hline$N=1$ & 1.7 & & & 2.6 & & \\
$N=2$ & 0.10 & 3.0 & & 1.7 & 3.1 & \\
$N=3$ & 0.10 & 1.73 & 3.0 & 1.0 & 1.3 & 1.8 \\
\hline \hline
\end{tabular}

scheme was used to fit two relaxation terms and a thermoviscous component to an approximate $\alpha_{k} \propto \omega^{\eta}$ power-law using Eq. (14). The relaxation frequency $\Omega_{\nu}$ was pre-set for the thermoviscous component, therefore leaving two $\Omega_{\nu}$ and three $\kappa_{\nu}$ parameters to be determined by the numerical scheme. The discrete relaxation frequencies and compressibility contributions are displayed in Table II

A fit for the same attenuation law to the fractional Zener model and to the corresponding continuous compressibility function is provided in Näsholm and Holm (2011) where Table II lists the parameters $\alpha, \tau_{\epsilon}, \tau_{\sigma}$, and $\kappa_{0}$.

\section{B. Power-law $\alpha_{k} \propto \omega^{1.1}$ for $f \in[100 \mathrm{kHz}, 1 \mathrm{GHz}]$}

In the following, the approach suggested in Section II.E is applied in a similar way as in Section III.A to construct power-law attenuation within the wider wave-frequency band $f \in[100 \mathrm{kHz}, 1 \mathrm{GHz}]$. This frequency band covers more or less all frequencies of conventional pulse-echo medical ultrasound imaging and microscopy. The properties of discrete relaxations with $\Omega_{\nu}$ evenly distributed in $\log \Omega$ were determined for distribution sets with 1 to 7 mechanisms. The attenuation functions corresponding to each relaxation mechanism set are displayed in Fig. 2.

\section{DISCUSSION AND CONCLUDING REMARKS}

As laid out in Section II.D the present paper demonstrates that for a continuous distribution of relaxation processes with the compressibility contribution distribution $\kappa_{\nu}(\Omega)$ populating the relaxation frequency range $\Omega \in\left[\Omega_{\mathrm{L}}, \Omega_{\mathrm{H}}\right]$, the effective wave equation for the wavefrequency $\omega$ satisfying $\Omega_{\mathrm{L}} \ll \omega \ll \Omega_{\mathrm{H}}$ is the same as for the relaxation processes populating the whole $\Omega \in[0, \infty]$. This work thus supports the intuitive conjecture that for a distribution of relaxation processes covering all frequencies, it is the $\Omega_{\nu}$ within the wave-frequency bandwidth that mainly contribute to the attenuation. The wave may thus be seen as probing the medium around the relaxation frequencies within and close to the wave-frequency bandwidth.

When instead $\omega \ll \Omega_{\mathrm{L}}<\Omega_{\mathrm{H}}$ or $\Omega_{\mathrm{L}}<\Omega_{\mathrm{H}} \ll \omega$, the effective wave equation becomes the same as for a single discrete NSW relaxation mechanism at $\Omega_{\nu}=\left(\Omega_{\mathrm{L}}+\Omega_{\mathrm{H}}\right) / 2$. 

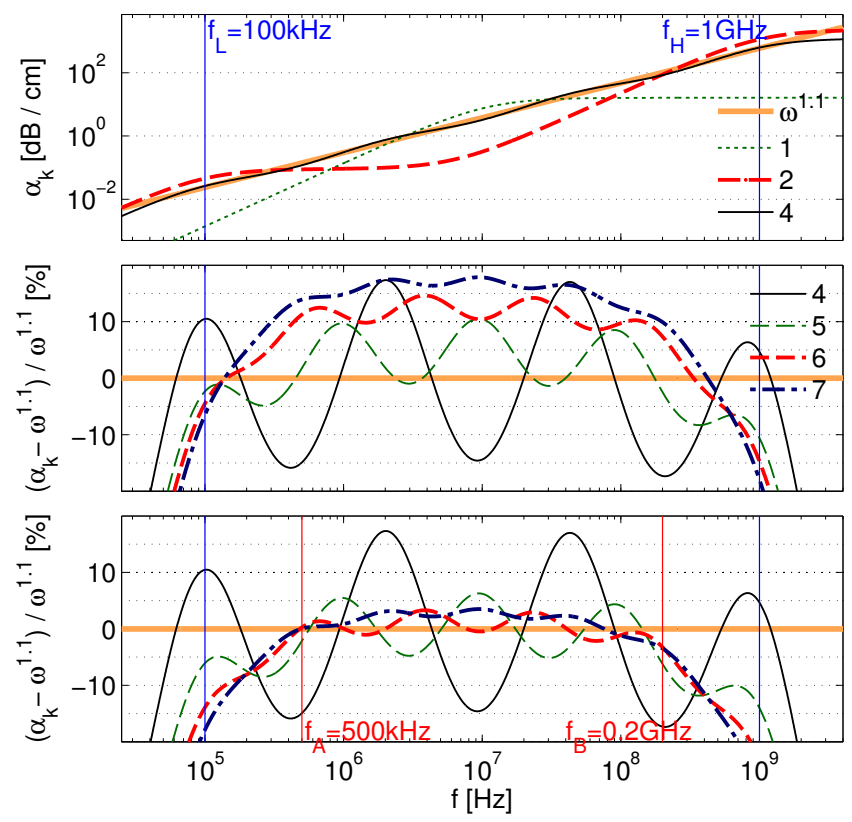

FIG. 2. (Color online) Attenuation as a function of frequency for $N$ relaxation mechanisms determined by the method proposed in Section II.E aiming at constructing a power-law $\propto \omega^{1.1}$ (thick solid line) valid within the wide interval between $100 \mathrm{kHz}$ and $1 \mathrm{GHz}$. The horizontal axes represent wavefrequency. Top pane: the attenuation functions for $N=1$ (thin dashed line), $N=2$ (thick dashed line), and $N=4$ (thin solid line). Middle pane: Relative difference between the attained relaxation attenuation functions and the powerlaw, with each attenuation normalized in order to make the minimum and maximum relative differences equal in magnitude within $100 \mathrm{kHz}$ and $1 \mathrm{GHz}$ for $N=4$ (thin solid line), $N=5$ (thin dashed line), $N=6$ (thick dashed line), and $N=7$ (thick dash-dotted line). Bottom pane: Same as the middle pane, however with the normalization taking the more narrow frequency interval between $500 \mathrm{kHz}$ and $0.2 \mathrm{GHz}$ into account.

All attenuation models that may be written as a superposition of NSW relaxations are causal.

In particular this work considers the Mittag-Leffler function related distribution case $\kappa_{\nu}^{\prime}(\Omega)$ as given in (20). For a fully populated range $\Omega \in[0, \infty]$ this has previously been shown to produce the four-parameter fractional Zener wave equation prompting three distinct frequency power-law attenuation regimes. If this relaxation mechanism distribution instead populates only $\Omega \in\left[\Omega_{\mathrm{L}}, \Omega_{\mathrm{H}}\right]$, as shown in Section II.D.2 the 4-parameter fractional Zener wave equation is still valid as long as the wave-frequency $\omega$ satisfies $\Omega_{\mathrm{L}} \ll \omega \ll \Omega_{\mathrm{H}}$. Actually, the distribution $\kappa_{\nu}^{\prime}(\Omega)$ may, as shown in Section II.C.2 for three different $\Omega$ regions be appropriately approximated by $\propto \omega^{d}$, with $-2<d \leq 0$. These regions are linked to the attenuation power-law frequency regimes of the 4-parameter fractional Zener wave equation.

The insights of Sections \I.C and II.D provide the basis upon which a proposed method for selection of discrete relaxation distribution properties relies. The discrete re- laxation parameter parameter selection approach is laid out in Section II.E, which is intended to be a key advance of the current paper. One may hence avoid applying numerical minimization methods to determine the relaxation parameters, which becomes cumbersome especially when there is a large number of mechanisms. This proposed selection of discrete parameters is model-based primarily in the sense that for a chosen process relaxation frequency $\Omega_{\nu}$, the compressibility $\kappa_{\nu}$ is decided from the distribution $\kappa_{\nu}^{\prime}\left(\Omega_{\nu}\right)$ given in (20). The $2 N$-parameter determination task to decide the $\Omega_{\nu}$ and $\kappa_{\nu}$ values for a model of $N$ relaxation mechanisms thus becomes an $\mathrm{N}$-parameter determination problem. However for determination of the $N$ relaxation frequency parameters, the straightforward choice to distribute $\Omega_{\nu}$ logarithmically equi-spaced within which $\Omega_{\nu} \in\left[\Omega_{\mathrm{L}}, \Omega_{\mathrm{H}}\right]$ is proposed here. The limits $\Omega_{\mathrm{L}}$ and $\Omega_{\mathrm{H}}$ are set similar to the maximum and minimum wave-frequencies where the attenuation model is to be valid.

As power-law attenuation is commonly encountered for large wave-frequency intervals in complex media, this work puts emphasis on the determination of discrete relaxation parameters to yield power-law attenuation within a given frequency interval. The theoretical considerations of Section $\prod$ are supplemented by two numerical examples in Section III. For the first example, where the attenuation is displayed in Fig. 1, we note that for 3 relaxation the chosen weighted mechanisms give rise to an attenuation differs by less than $14 \%$ relative to the pure power-law $\alpha_{k}(\omega) \propto \omega^{1.1}$ within the whole frequency region of interest $f \in[100 \mathrm{kHz}, 30 \mathrm{MHz}]$. For two mechanisms, the highest relative difference is $63 \%$. By contrast, the Yang and Cleveland (2005) weighted sum of three relaxations corresponds to a maximum relative difference in $\alpha_{k}(\omega)$ of $68 \%$. When normalizing the Yang and Cleveland attenuation, the maximum difference is reduced to $29 \%$, which is however still more than double the $14 \%$ limit attained when setting the parameters following the prescription suggested in the current paper for the same number of relaxation mechanisms. Judging from the maximum relative absolute difference between the resulting $\alpha_{k}(\omega)$ and the pure power-law with the exponent 1.1, the proposed method hence is more advantageous.

Inspection of the resulting attenuation curves of Fig. 1 hints the possibility of attaining less relative difference between $\alpha_{k}(\omega)$ and the power-law by straightforward adjustment of the width of the populated relaxation frequency region. This possibility is now briefly explored. For low $N$, it seems appropriate to make the populated region smaller, while for larger $N$ the relative difference between attained $\alpha_{k}(\omega)$ and the wanted power-law increases close to $\Omega_{\mathrm{L}}$ and $\Omega_{\mathrm{H}}$ hence suggesting to make the populated frequency region wider. For $N=2$, shrinking the populated relaxation frequency range from $\Omega \in[0.1,30] \mathrm{MHz}$ via $[0.2,15] \mathrm{MHz}$ down to $[0.3,11] \mathrm{MHz}$ decreases the maximum relative difference from $63 \%$ via $39 \%$ down to $28 \%$ (see Fig. 31), which is actually about the same as was attained for the normalized Yang and Cleveland fit for three relaxation processes.

The second numerical example for which the resulting 


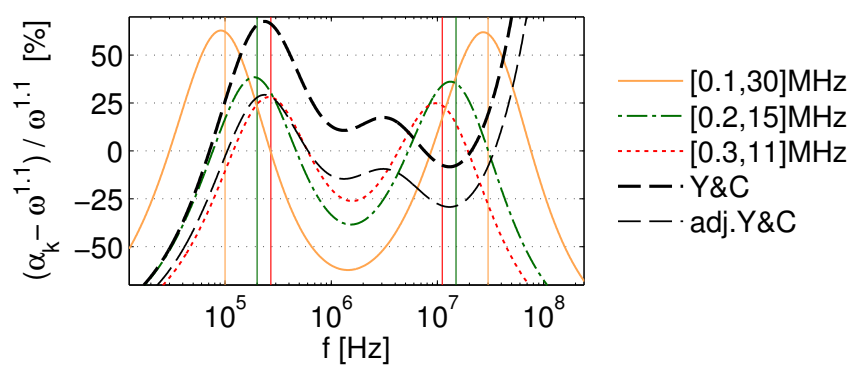

FIG. 3. (Color online) Relative difference between the attained attenuation and the wanted power-law $\propto \omega^{1.1}$ for $N=2$ mechanisms, as attained when the relaxation frequencies are $\Omega_{1,2}=\{0.1,30\} \mathrm{MHz}$ (solid line), $\{0.2,15\} \mathrm{MHz}$ (dash-dotted line), and $\{0.3,11\} \mathrm{MHz}$ (dotted line). All these attenuation functions are normalized so that absolute maximum and absolute minimum of the relative difference are equal. For reference, the Yang and Cleveland (2005) attenuation is also included, both in the original form (thick dashed line) and in the adjusted normalized form (thin dashed line). The vertical lines indicate frequencies of the explored relaxation mechanisms.

attenuation functions are displayed in Fig. 2 illustrates that that for as few as five discrete relaxation processes, an adequate power-law differing by less than $11 \%$ from the wanted power-law may be constructed within the very wide frequency band $f \in[100 \mathrm{kHz}, 1 \mathrm{GHz}]$.

The fractional Zener compressibility of Eq. (15) is for $\alpha=\beta$ similar to the Cole-Cole expression for complex dielectric permittivity, which is empirically shown to be valid in a variety of complex media. A single discrete acoustical NSW relaxation mechanism in the dielectric permittivity context corresponds to a single Debye term. The adjustment and weighting of multiple discrete Debye terms to emulate Cole-Cole dielectrical behavior is treated e.g. in Rekanos and Papadopoulos (2010), where a Padé approximant approach is applied, in Tofighi (2009) where an error-minimization method is employed choose the relaxation times at which the Cole-Cole relaxation distribution is sampled, in Kellev et al. (2007) where the relaxation frequencies are found using a nonlinear method and the weights are found using a linear least squares approach, as well as in Clegg and Robinson (2010) where a genetic algorithm is applied to find the multiple Debye parameters. The parameter determination approach brought forward in the present paper should also be tested out for selection of a discrete set of Debye terms to emulate the Cole-Cole dielectric permittivity.

The model applied in Berkhoff et al. (1996) was contextualized in the discussion of Nsholm and Holm (2011). It was found that it corresponds to band-limited continuous relaxations with compressibility contributions given by $\kappa_{\nu}(\Omega) \propto \Omega^{-1}$.

We note that based on experimental evidence, Jongen et al. (1986) suggests the attenuation for example in beef liver to be $\propto \omega^{2}$ below a certain cut-off frequency $\omega_{c}$ and $\propto \omega$ at higher frequencies. Such behavior is attained by the band-limited continuous relaxation distribution framework of the present work when the lowest frequency $\Omega_{\mathrm{L}}$ of the populated relaxation frequency band is equal to $\omega_{c}$.

The $N$ relaxation parameter determination method of Section II.E corresponds to mapping a time-fractional wave equation into an integer-order one of highest order $N+2$ with the intent of the mapping being adequately valid within a given wave-frequency band. Because fractional-order differential wave equations require extra care in simulations, the method suggested here hence has a potential to facilitate numerical wave-propagation calculations. Comparison to previously suggested such numerical schemes as e.g. Wismer and Ludwig (1995); Caputo et al. (2011), is an appropriate future work connected to this paper.

From a practical point of view the method of this paper to determine discrete relaxation parameters is appealing because the anomalous physics of fractional-order differential equations is converted to a differential equation with an finite set of higher-order integer derivatives. This conversion is tailored for the frequency bandwidth of interest. For such conversions to be valid for all frequencies, an infinite number of terms are needed in the integerorder differential equation. Thence the narrowing of the frequency region is traded off into the convenience of having a finite highest derivative order.

Finally this work calls upon further experimental verifications and more profound theoretical connection between the relaxation models and fundamental physical properties of complex materials. Hopefully the links between fractional calculus, observed attenuation behaviors, the stunningly common power-law patterns of nature, and the micromechanical structure of matter are to be further clarified.

\section{Acknowledgments}

The author would like to thank Prof. Sverre Holm for interesting discussions and valuable advice. This research was partly supported by the "High Resolution Imaging and Beamforming" project of the Norwegian Research Council.

Adolfsson, K., Enelund, M., and Olsson, P. (2005). "On the fractional order model of viscoelasticity", Mech. Time-Dep. Mater. 9, 15-34.

Ainslie, M. and McColm, J. G. (1998). "A simplified formula for viscous and chemical absorption in sea water", J. Acoust. Soc. Am. 103, 1671-1672.

Bagley, R. L. and Torvik, P. J. (1983). "Fractional calculus A different approach to the analysis of viscoelastically damped structures", AIAA J. 21, 741-748.

Bass, H., Sutherland, L., Zuckerwar, A., Blackstock, D., and Hester, D. (1995). "Atmospheric absorption of sound: Further developments", J. Acoust. Soc. Am. 97, 680-683.

Bateman, H. and Erdélyi, A. (1954). Tables of integral transforms, volume 1, chapter 6 (McGraw-Hill, New York). Berkhoff, A. P., Thijssen, J. M., and Homan, R. J. F. (1996). "Simulation of ultrasonic imaging with linear arrays in causal absorptive media", Ultrasound Med. Biol. 22, 245-259. 
Caputo, M., Carcione, J. M., and Cavallini, F. (2011). "Wave simulation in biologic media based on the Kelvin-Voigt fractional-derivative stress-strain relation", Ultrasound Med. Biol. 37, 996-1004.

Clegg, J. and Robinson, M. P. (2010). "A genetic algorithm used to fit Debye functions to the dielectric properties of tissues", in IEEE Congress on Evolutionary Computation, 1-8. Glöckle, W. G. and Nonnenmacher, T. F. (1991). "Fractional integral operators and Fox functions in the theory of viscoelasticity", Macromolecules 24, 6426-6434.

Holm, S. and Näsholm, S. P. (2011). "A causal and fractional all-frequency wave equation for lossy media", J. Acoust. Soc. Am. 130, 2195-2202.

Holm, S. and Sinkus, R. (2010). "A unifying fractional wave equation for compressional and shear waves", J. Acoust. Soc. Am. 127, 542-548.

Jongen, H. A. H., Thijssen, J. M., van den Aarssen, M., and Verhoef, W. A. (1986). "A general model for the absorption of ultrasound by biological tissues and experimental verification", J. Acoust. Soc. Am. 79, 535-540.

Kelley, D. F., Destan, T. J., and Luebbers, R. J. (2007). "Debye function expansions of complex permittivity using a hybrid particle swarm-least squares optimization approach", IEEE Trans. Antennas Propag. 55, 1999-2005.

Kelly, J. F. and McGough, R. J. (2009). "Fractal ladder models and power law wave equations", J. Acoust. Soc. Am. 126, 2072-2081.

Liebler, M., Ginter, S., Dreyer, T., and Riedlinger, R. E. (2004). "Full wave modeling of therapeutic ultrasound: Efficient time-domain implementation of the frequency power-law attenuation", J. Acoust. Soc. Am. 116, 2742-2750.

Nachman, A. I., Smith III, J. F., and Waag, R. C. (1990). "An equation for acoustic propagation in inhomogeneous media with relaxation losses", J. Acoust. Soc. Am. 88, 15841595 .

Näsholm, S. P. and Holm, S. (2011). "Linking multiple relaxation, power-law attenuation, and fractional wave equations", J. Acoust. Soc. Am. 130, 3038-3045.

Newman, M. E. J. (2005). "Power laws, Pareto distributions and Zipf's law", Contemp. Phys 46, 323-351.

Papoulia, K., Panoskaltsis, V., Kurup, N., and Korovajchuk, I. (2010). "Rheological representation of fractional order viscoelastic material models", Rheol. Acta 49, 381-400.

Pauly, H. and Schwan, H. P. (1971). "Mechanism of absorption of ultrasound in liver tissue", J. Acoust. Soc. Am. 50, 692-699.

Prieur, F. and Holm, S. (2011). "Nonlinear acoustic wave equations with fractional loss operators", J. Acoust. Soc. Am. 130, 1125-1132.

Prieur, F., Vilenskiy, G., and Holm, S. (2012). "A more fundamental approach to the derivation of nonlinear acoustic wave equations with fractional loss operators", J. Acoust. Soc. Am. 132, 2169-2172.

Rekanos, I. T. and Papadopoulos, T. G. (2010). "FDTD modeling of wave propagation in Cole-Cole media with multiple relaxation times", IEEE Antenn. Wireless Propag. Lett. 9, 67-69.

Roitner, H., Bauer-Marschallinger, J., Berer, T., and Burgholzer, P. (2012). "Experimental evaluation of time domain models for ultrasound attenuation losses in photoacoustic imaging", J. Acoust. Soc. Am. 131, 3763-3774.

Rossikhin, Y. A. and Shitikova, M. V. (2001). "Analysis of rheological equations involving more than one fractional parameters by the use of the simplest mechanical systems based on these equations", Mech. Time-Depend. Mat. 5, 131-175.

Samko, S. G., Kilbas, A. A., and Marichev, O. I. (1993). Frac- tional Integrals and Derivatives: Theory and Applications, chapter 2 (Gordon and Breach, New York).

Szabo, T. L. and Wu, J. (2000). "A model for longitudinal and shear wave propagation in viscoelastic media", J. Acoust. Soc. Am. 107, 2437-2446.

Tabei, M., Mast, T. D., and Waag, R. C. (2003). "Simulation of ultrasonic focus aberration and correction through human tissue", J. Acoust. Soc. Am. 113, 1166-1176.

Tofighi, M.-R. (2009). "FDTD modeling of biological tissues Cole-Cole dispersion for $0.5-30 \mathrm{GHz}$ using relaxation time distribution samples - novel and improved implementations", IEEE Trans. Microw. Theory Tech. 57, 2588-2596.

Treeby, B. E., Jaros, J., Rendell, A. P., and Cox, B. T. (2012). "Modeling nonlinear ultrasound propagation in heterogeneous media with power law absorption using a $k$-space pseudospectral method", J. Acoust. Soc. Am. 131, 4324-4336.

Vilensky, G., ter Haar, G., and Saffari, N. (2012). "A model of acoustic absorption in fluids based on a continuous distribution of relaxation times", Wave Motion 49, 93-108.

Widder, D. V. (1966). "A transform related to the poisson integral for a half-plane", Duke Math. J. 33, 355-362.

Wismer, M. G. (2006). "Finite element analysis of broadband acoustic pulses through inhomogenous media with power law attenuation", J. Acoust. Soc. Am. 120, 3493-3502.

Wismer, M. G. and Ludwig, R. (1995). "An explicit numerical time domain formulation to simulate pulsed pressure waves in viscous fluids exhibiting arbitrary frequency power law attenuation", IEEE Trans. Ultrason. Ferroelectr., Freq. Control 42, 1040-1049.

Yang, X. and Cleveland, R. O. (2005). "Time domain simulation of nonlinear acoustic beams generated by rectangular pistons with application to harmonic imaging", J. Acoust. Soc. Am. 117, 113-123. 